\title{
CARACTERIZACIÓN DE LA PRODUCCIÓN DE FRIJOL EN LA PROVINCIA DE COTOPAXI ECUADOR: CASO COMUNA PANYATUG
}

\section{CHARACTERIZATION OF PRODUCTION OF BEAN IN ECUADOR COTOPAXI PROVINCE: CASE COMMUNE PANYATUG}

\author{
Emma Torres Navarrete ${ }^{1,3}$, David Quisphe Caiza ${ }^{2}$, Adolfo Sánchez Laíño ${ }^{1,3}$, Mariana Reyes Bermeo², Betty \\ González Osorio $^{1,4}$, Alexandra Torres Navarrete ${ }^{5}$, Alex Cedeño Briones ${ }^{2}$, Alexandra Haro Chong ${ }^{2}$ \\ ${ }^{1}$ Dirección de Investigación Científica y Tecnológica, Universidad Técnica Estatal de Quevedo. Campus Finca Experimental \\ "La María", km 7 vía Quevedo-El Empalme. C. P. 73. Mocache, Los Ríos, Ecuador. \\ ${ }^{2}$ Unidad de Estudios a Distancia, Universidad Técnica Estatal de Quevedo. Campus Ing. Manuel Haz Álvarez, $\mathrm{km} 1.5$ vía a \\ Santo Domingo de los Tsáchilas. C. P. 73. Quevedo, Los Ríos, Ecuador. \\ ${ }^{3}$ Facultad de Ciencias Pecuarias, Universidad Técnica Estatal de Quevedo. Campus Finca Experimental "La María”, km 7 vía \\ Quevedo-El Empalme. C. P. 73. Mocache, Los Ríos, Ecuador. \\ ${ }^{4}$ Facultad de Ciencias Empresariales, Universidad Técnica Estatal de Quevedo. Campus Ing. Manuel Haz Álvarez, km 1.5 vía \\ a Santo Domingo de los Tsáchilas. C. P. 73. Quevedo, Los Ríos, Ecuador. \\ ${ }_{5}^{5}$ Universidad Estatal Amazónica, Carrera de Ingeniería Agropecuaria, Campus principal, $\mathrm{km} .2$ 1/2 vía Napo (paso lateral) \\ Puyo - Pastaza
}

Emails: etorres@uteq.edu.ec; jenidasan@hotmail.com; arsanchez@uteq.edu.ec; mreyes@uteq.edu.ec; bgonzalez@uteq.edu.ec; atorres@uea.edu.ec; acedeño@uteq.edu.ec; aharo@uteq.edu.ec

\begin{abstract}
RESUMEN
E lobjetivo del trabajo fue obtener información sobre Lla producción, comercialización y rentabilidad del frijol canario en la Comuna Panyatug del cantón Pangua, provincia de Cotopaxi-Ecuador en el año 2009 , con la finalidad de conocer e informar a los productores las fortalezas y amenazas en esta actividad productiva. Para ello se aplicó un muestreo aleatorio simple a 64 productores de frijol, 3 acopiadores rurales y 3 comerciantes mayoristas. Se obtuvieron los costos de producción y rentabilidad así como los márgenes del productor, comercialización, acopiador rural. Los resultados demuestran que los productores tienen experiencia en cultivar el frijol de 5 a 48 años; la superficie dedicada al frijol varía de 0.37 a 5 ha; mantienen una economía de subsistencia y en sus sistemas de producción utilizan $97 \%$ de la mano de obra familiar, $100 \%$ semilla reciclada, no controlan plagas ni enfermedades y poseen otros cultivos como cebada, mora, trigo y arveja para venta y autoconsumo. Los costos de producción corresponden a 1,172 USD $\mathrm{ha}^{-1}$, la producción media es $725.76 \mathrm{~kg} \mathrm{ha}^{-1}$, el precio de venta promedio 1.675 USD el kg seco, generando una $\mathrm{R}$ B/C del $36.18 \%$ en 7 meses del ciclo productivo. Los márgenes de comercialización indican que por cada 1 dólar pagado por el consumidor corresponde al productor 0.44 USD y a la intermediación 0.56 USD, ésta última se distribuye para el acopiador rural 0.029 USD, el mayorista comisionista 0.225 USD y para el detallista 0.306 USD.
\end{abstract}

Palabras clave: Productores de frijol, producción, rentabilidad, márgenes de comercialización.

Recibido: 21-Enero-2013. Recibido en forma corregida: 27-Febrero-2013. Aceptado: 28-Marzo-2013.

Publicado como ARTÍCULO CIENTÍFICO en Ciencia y Tecnología 6(1): 23-31.

Enero - Junio de 2013

ISSN 1390-4051 impreso; ISSN 1390-4043 electrónico

\section{Abstract}

$\mathrm{T}$ he purpose of this study was to obtain information about production, profitability and commercialization from canary beans in Payatung comuna of the Pangua canton in Cotopaxi - Ecuador in 2009, the aim was to detect strengths and threats about this activity and explain to the farmers producers. It was applied a random sampling to 64 beans producers, 3 rural collectors and 3 wholesalers. It was obtained profitability, production costs, and income to farmers. The research showed that has an experience about 5 to 48 years in bean, the surface of production can be from $0.37 \mathrm{ha}$ to $5.0 \mathrm{ha}$; the subsistence economy is provided by its own manpower, are used seeds recycled $100 \%$, the pests and diseases not are controlled and to sales and auto consumption they have crops as berry, peas, corn and barley. The production cost are 1,172 USD ha-1, the half of production is $725.76 \mathrm{~kg} \mathrm{ha}^{-1}$, the sales price is 1.675 USD el $\mathrm{kg}$, obtaining a $\mathrm{R} \mathrm{B} / \mathrm{C}$ del $36.18 \%$ productive cycle in 7 months, the trading margin indicate that for each 1 dollar paid for the consumer correspond 0.44 USD to the farmer producer, and the intermediaries $0.56 \mathrm{USD}$, the intermediaries are distributed 0.029 USD to rural collector, 0.225 USD wholesaler, and 0.306 USD to retail.

Key words: Bean producers, production, profitability, marketing margins. 


\section{INTRODUCCIÓN}

$\mathrm{E}$ 1 frijol (Phaseolus vulgaris L.), presuntamente, fue introducido en América por las tribus nómadas que cruzaron el estrecho de Bering hasta Alaska. Hay evidencias que en el siglo X los Aztecas en México usaron el fríjol como un grano básico, y que los Incas lo introdujeron a Suramérica (Velásquez y Giraldo, 2005).

Es la leguminosa de grano de consumo humano directo más importante en el planeta; ocupa el octavo lugar entre las leguminosas sembradas en el mundo. Para la población ecuatoriana constituye una de las principales fuentes de proteína y carbohidratos.

Mundialmente el frijol es la leguminosa alimenticia más importante para cerca de 300 millones de personas, que, en su mayoría, viven en países en desarrollo, debido a que este cultivo, conocido también como "la carne de los pobres", es un alimento poco costoso para consumidores de bajos recursos. El frijol es especialmente importante en la alimentación de mujeres y niños; además, tiene gran importancia económica, pues genera ingresos para millones de pequeños agricultores (Velásquez y Giraldo, 2005).

Es una especie de clima templado y por tanto, no crece bien en zonas frías o calientes. El umbral térmico de la especie es de $10^{\circ} \mathrm{C}$ como temperatura mínima y $25^{\circ} \mathrm{C}$ como temperatura máxima. Las heladas producen la muerte de las plantas, en tanto que el exceso de calor afecta a la floración y aumenta la esterilidad de las flores. Las características del ciclo vegetativo dependen de la acumulación de temperaturas. Los cultivares adaptados a las zonas más cálidas tienen un ciclo de alrededor de 90 días, mientras que los adecuados a zonas más frescas llegan a tenerlo hasta 250 días (Erazo, 2005).

A nivel mundial se producen 18.991,954 t, siendo los mayores productores mundiales: Brasil (3 millones de t), India (2.9 millones de t), México (1.5 millones de t) Nicaragua, Myanmar (1.9 millones t), China (1.9 millones $t$ ) entre otros países. Ecuador produce 39,725 $\mathrm{t}$, es decir, el $0.2 \%$ de la producción mundial (SICAMAG, 2000).

En el Ecuador el frijol es la leguminosa de mayor área de cultivo y consumo (Garver et al., 2008) actualmente se cosecha 89,789 hectáreas de las 105,127 ha, sembradas de esta leguminosa en grano seco y 15,241 ha en verde o tierno de las 16,464 ha, sembradas (SICA-MAG-INEC, 2002). El rendimiento promedio de frijol registrado en Ecuador es bajo, $430 \mathrm{~kg} \mathrm{ha}^{-1}$ en monocultivo y $110 \mathrm{~kg} \mathrm{ha}^{-1}$ cuando está asociado con maíz (INEC, 2002), frente al rendimiento potencial del cultivo que sobrepasa los 2,000 $\mathrm{kg} \mathrm{ha}^{-1}$ (Sanders y Schwartz, 1980). Los bajos rendimientos han sido asociados a enfermedades foliares y a estreses abióticos como sequía y baja fertilidad de suelos. Sin embargo, las enfermedades foliares son las responsables de las mayores pérdidas de producción en las áreas más importantes de cultivo del país. Las principales enfermedades que atacan al cultivo de frijol en Ecuador son: roya (Uromyces appendiculatus), antracnosis (Colletotrichum lindemuthianum) y mancha angular (Phaeoisariopsis griseola) (Murillo et al., 2005). Otro factor al que se atribuyen los bajos rendimientos es el uso de semilla reciclada, debido a que tienen poco acceso a materiales genéticos, porque en la zona no existe un mercado formal de variedades mejoradas de frijoles. Las semillas criollas son susceptibles a plagas y enfermedades, y tienen bajo potencial de rendimiento (Mendoza, 1998; citado por Galindo-González y Zandate-Hernández, 2006).

Según técnicos del Ministerio de Agricultura y Ganadería (elcomercio.com, 2011) en el país hay 35,000 hectáreas sembradas de este grano, la Sierra Norte, con 8,000 hectáreas, es la zona de mayor producción del grano en el país. Aunque existen 50 especies de frijol, las variedades rojo moteado, canario, calima negro y blanco panamito son las más apetecidas por el mercado. El frijol canario es conocido como el rey de los frijoles por su sabor y textura. Los granos son grandes o medianos, semirredondos y de color amarillo. Hay varias especies parecidas que poseen tonos amarillentos. Entre los principales están el azufrado, el canario y el garbancillo.

Según PROFRIZA (2000) el 95\% del frijol que se cultiva en el Ecuador, se cultiva en las provincias de la Sierra, las siembras se ubican entre los 1,000 y 2,800 metros sobre el nivel del mar, con una amplia variación de tipos de suelo, sistemas de cultivo, condiciones climáticas y manejo agronómico y problemas de producción y rendimientos unitarios.

Los principales productores de frijol, en países de Latinoamérica y África, son pequeños agricultores cuyas áreas de cultivo son menores a una hectárea. La producción está fundamentalmente destinada para autoconsumo y venta en mercados locales (Pachico, 1989), además dentro de sus sistemas de producción existe un alto uso de mano de obra sea ésta familiar o contratada (González et al., 2006). Esto demuestra la importancia económica, nutricional y social del cultivo para los agricultores de bajos recursos, debido a los ingresos financieros que provee la venta de la producción, así como por contribuir a la seguridad alimentaria de la población de estas zonas. El consumo per capita de frijol puede variar de un país a otro o de una región a otra región dentro de un mismo país. El estatus social y económico de las familias también influye en las preferencias de consumo (Singh, 1999). Frecuentemente, el consumo de frijol es mayor en familias de bajos ingresos que viven en zonas rurales y urbanas. En Latinoamérica, el mayor consumo per 
capita se registra en Brasil y México con más de 13 kilogramos por año. En países de África Central y Oriental (Ruanda y Burundi) el consumo por persona es mayor a $40 \mathrm{~kg}$, en Ecuador, el consumo de frijol por persona es de $4 \mathrm{~kg}$ (FAO, 2005), un valor relativamente bajo si se lo compara con otros países de Latinoamérica. Sin embargo, el consumo por persona al año puede superar los $40 \mathrm{~kg}$ en las zonas de mayor producción de frijol del país, como sucede en los valles del Chota y Mira (PRONALEG-GA, 2005).

El frijol canario es de suma importancia para los habitantes de la comuna Panyatug perteneciente al cantón Pangua, provincia de Cotopaxi, ya que además de ser un producto básico de la canasta familiar de los habitantes tiene un alto valor nutritivo (Trujillo, 2007), pues contiene proteína (18-25\%), fibra (18\%), grasa $(1.7 \%)$, carbohidratos $(61.4 \%)$ vitaminas $\mathrm{y}$ minerales. Además es un gran generador de empleo entre las familias campesinas de la zona debido a que generalmente toda la familia se dedica a dicha actividad. Se conoce que la comuna es eminentemente frijolera, pero no existen datos que respalden información de tipo socioeconómico de la zona, se desconoce los sistemas de producción, los niveles de producción y el sistema de comercialización del grano, por lo que se plantearon los siguientes objetivos: Caracterizar los sistemas de producción del frijol canario, identificar y cuantificar los canales, los participantes y los márgenes de comercialización del producto $\mathrm{y}$, determinar la rentabilidad de la producción de frijol canario en la Comuna Panyatug del Cantón Pangua.

\section{Materiales y Métodos}

$\mathrm{E}^{1}$ estudio se realizó en la Provincia de CotopaxiEcuador, cantón Pangua, parroquia matriz El
Corazón, comuna Panyatug ubicada sobre los 1800 msnm, con una temperatura promedio de $18^{\circ} \mathrm{C}$, humedad relativa de $12 \%$. Las coordenadas geográficas son: $1^{\circ} 12^{\prime} 0^{\prime \prime}$ de latitud sur y $79^{\circ} 6^{\prime} 0^{\prime \prime}$ de longitud oeste (Figura 1).

La estrategia que se utilizó para la ejecución de la investigación fue la técnica de la encuesta directa a productores de frijol canario, con estructuración de tipo cuanti-cualitativo y una dimensión temporal de tipo transversal, es decir, los datos se recolectaron en un solo corte de tiempo.

Para determinar el tamaño de la muestra se aplicó el muestreo aleatorio simple, sobre el cual todos y cada uno de los individuos de la población tienen la misma e independiente probabilidad de ser seleccionados como miembros de la muestra (Santoyo-Cortés et al., 2000).

La ecuación aplicada fue:

$$
n=\frac{(N)\left(Z^{2} \infty / 2\right)(p n)(q n)}{(N-1)\left(d^{2}\right)+\left(Z^{2} \infty / 2\right)(p n)(q n)}
$$

Donde:

$\mathrm{n}=$ tamaño de la muestra;

$\mathrm{N}=600$ (población total o universo);

$\mathrm{d}=10 \%$ (error muestral);

$\mathrm{Z} 2 \propto / 2=1.69$ (Coeficiente de confianza o confiabilidad); pn $=0.5$ (probabilidad de éxito o aceptación);

$\mathrm{qn}=0.5$ (probabilidad de fracaso o rechazo).

Esta ecuación tiene las siguientes características: es aplicable cuando no se conoce pn y qn; mediante el cálculo de "n" a través de ésta, se obtiene un tamaño máximo que cubre cualquier otro posible tamaño, y los resultados obtenidos son de absoluta confianza; ésta es susceptible de utilizarse como una estimación del tamaño de muestras para encuestas o muestreos piloto,

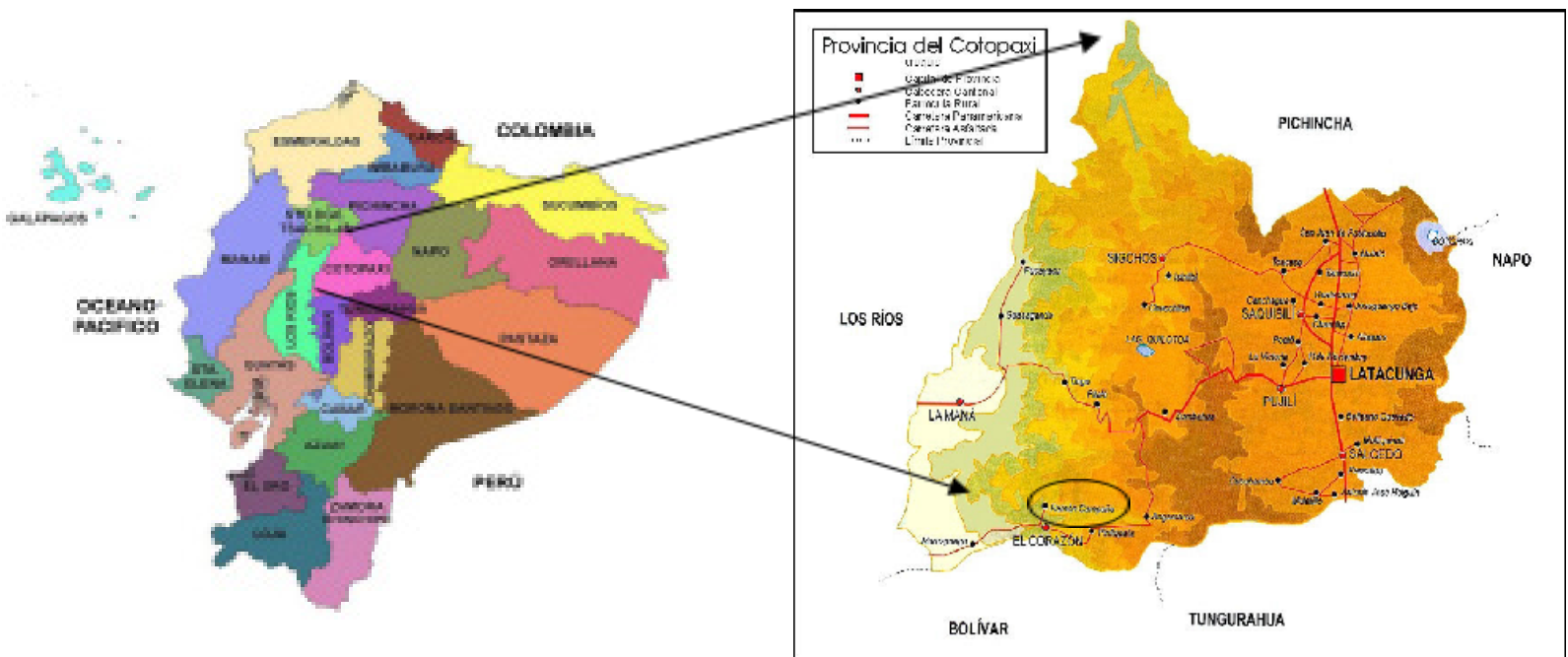

Figura 1. Ubicación de la zona de estudio: Cantón Pangua, provincia de Cotopaxi 
eliminando tamaños arbitrarios.

Al aplicar la fórmula señalada, se determinó que $n=64$. La selección de las unidades de muestreo se realizó extrayendo aleatoriamente una a una las unidades de la población, por medio de tablas de números aleatorios.

Para recopilar la información necesaria, se diseñó, probó y aplicó un cuestionario, tomando en consideración información requerida, tipo y contenido de preguntas, forma de respuesta, palabras a utilizar y su secuencia, y prueba de cuestionario. Las preguntas fueron de hecho y de opinión; las respuestas, abiertas, cerradas y dicotómicas.

Le encuesta aplicada no fue muy extensa, se consideraron en este trabajo aquellas variables que se relacionan con la toma de decisiones para el cultivo de frijol. Dichas variables se agruparon a su vez en los siguientes aspectos: socioeconómicos (Edad del productor, años dedicados a la actividad, integrantes del núcleo familiar, escolaridad), sistemas de producción (Uso de variedades, fitosanidad, fertilización), rentabilidad (Costos, producción, ingresos) y comercialización del grano (Sistema de venta, precios del productor del comerciante y del consumidor final).

La información se analizó mediante el uso de Statistical Package for the Social Sciences (SPSS) que es especializado en estudios sociales y mercadotécnicos (Microsoft, 1999).

Para determinar los márgenes de comercialización se entrevistó a tres acopiadores rurales y tres mayoristas que realizan actividades de comercialización de frijol en la zona. Se recopiló información referente a sus funciones y precios de compra y venta. Una vez identificados los comerciantes se calcularon los márgenes de comercialización de acuerdo a la metodología propuesta por Espinosa et al. (2008). Para calcular estos indicadores se aplicaron las siguientes formulas:

\section{Margen Bruto de comercialización (MBC)}

$M B C=\frac{\text { Precio del consumidor }- \text { Precio del agricultor }}{\text { Precio del consumidor }} \times 100$

\section{Margen del productor (PDP)}

$P D P=\frac{\text { Precio del consumidor }-M B C}{\text { Precio del consumidor }} \times 100$

Margen neto de comercialización (MNC)

MNC $=\frac{\text { Margen bruto de comercialización }- \text { Costos de mercadeo }}{\text { Precio pagado por el consumidor }} \times 100$

Margen bruto de comercialización del acopiador rural (MBC-AR)

$M B C-A R=\frac{\text { Precio de venta }- \text { Precio de compra }}{\text { Precio pagado por el consumidor }} \times 100$

\section{Margen bruto de comercialización del mayorista} (MBC-M)

$M B C-M=\frac{\text { Precio de venta }- \text { Precio de compra }}{\text { Precio pagado por el consumidor }} \times 100$

Los precios de compra y venta son los que paga el mayorista al acopiador rural y el minorista al mayorista

\section{Análisis económico}

Para el estudio de la rentabilidad de la producción de frijol, se analizaron los costos y los ingresos, a través de la relación beneficio/costo y la rentabilidad. Se calculó la estructura de costos de los diferentes rubros agrupados en directos e indirectos que utilizan los productores. Además, se calculó el costo y el margen de utilidad por kilo de frijol producido.

\section{Rentabilidad}

El análisis de rentabilidad económica se calculó con base a la relación beneficio/costo, la relación beneficio costo es un método que consiste en contrarrestar los beneficios obtenidos con los gastos generados durante el proceso de producción y se obtiene mediante la fórmula (Ruíz, 1996; citado por Villegas et al., 2009).

Rentabilidad $=\frac{\text { Beneficio } \text { Neto }}{\text { Costo de producción }} \times 100$

\section{Resultados y Discusión}

\section{Aspectos socioeconómicos}

El núcleo familiar de los productores encuestados está formado en promedio por 5 personas, siendo el rango inferior 1 y el superior 14 personas; de los cuales el promedio de personas adultas que conforman el hogar corresponde a 3 con rangos que van de 1 a 9 , mientras que la cantidad de niños en cada hogar es en promedio 2 con un rango que va de 0 a 7 niños.

Se determinó que el $72 \%$ de los productores son propietarios comunales de sus tierras, un 3\% son arrendatarios y un $25 \%$ de los encuestados cultivan terrenos de familiares o son terrenos prestados determinándose que un $28 \%$ son productores que no poseen tierras. Un gran porcentaje de productores entrevistados tienen más de 42 años $(82 \%)$, y existe un $14.3 \%$ de analfabetismo, mientras que un $79.6 \%$ tienen instrucción primaria. Los años dedicados a 
la producción de frijol por parte de los productores estudiados corresponde a un promedio de 22 años, con rangos que van de 5 a 48 años, lo cual evidencia que existe experiencia en el manejo del cultivo.

Las fincas de los productores tienen superficies promedios de 3.70 ha. Las superficies de terreno dedicada al cultivo de frijol corresponde a un promedio de 1.48 ha, siendo el rango inferior 0.37 ha y el rango superior 5 ha (Figura 2), esta información permite identificarlos como pequeños productores debido a que su sistema de producción está circunscrito a pequeñas propiedades generalmente terrenos escarpados.

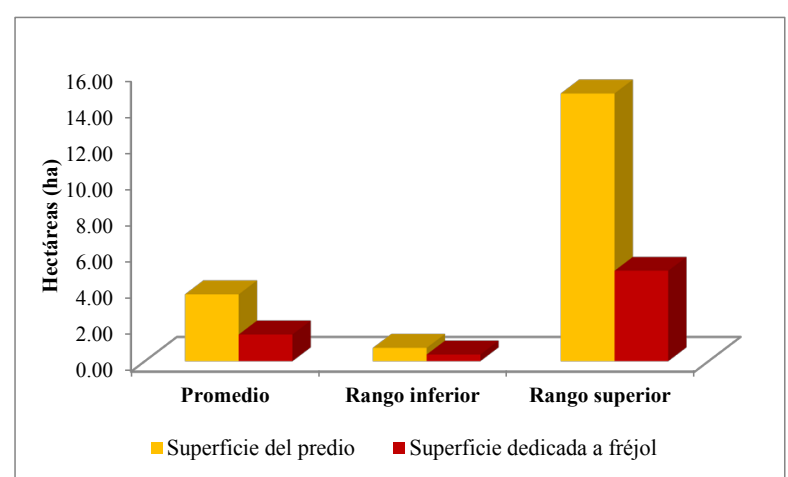

Figura 2. Superficie de las fincas y superficie dedicada al cultivo de frijol en la comuna Panyatug

Referente a otros rubros de producción explotados por los productores de la comuna Panyatug se determinó que el $98.44 \%$ de ellos cultiva maíz, el $53.14 \%$ también tiene en sus predios trigo y arveja, el $37.60 \%$ cultiva mora, el $29.69 \%$ cultiva cebada como medio de obtener ingresos además, un $48.44 \%$ de los productores manifestaron cultivar en mínimas cantidades durante todo el año arvejas, habas, y hortalizas, tanto para autoconsumo como para la venta (Cuadro 1). Cabe indicar que el cultivo de frijol es de suma importancia para estas familias no solo por representar ingresos económicos sino por la costumbre de consumir dicho producto en la dieta familiar, consumo que representa un total de $4.36 \pm 1.3 \mathrm{~kg}$ por persona año, este promedio es superior al reportado por PRONALEG-GA (2005) quienes indican que el consumo de frijol en Ecuador corresponde a $4 \mathrm{~kg}$ por persona año.

Un $3 \%$ de los productores se dedican exclusivamente a trabajar sus tierras, mientras que el $97 \%$ manifestaron que realizan actividades extra finca para complementar sus ingresos, entre estas actividades la más representativa corresponde a jornales en fincas productoras de licor y panela, o a su vez realizan trabajos puntuales como ayudantes de mecánica u otros oficios artesanales
.Cuadro 1. Otros cultivos que poseen los productores dedicados al cultivo de frijol en la comuna Panyatug

\begin{tabular}{lcr}
\hline Cultivos & $\begin{array}{c}\text { Frecuencia } \\
\text { Absoluta }\end{array}$ & $\begin{array}{c}\text { Frecuencia } \\
\text { Relativa }\end{array}$ \\
\hline Maíz & 63 & 98.44 \\
Trigo & 34 & 53.13 \\
Arveja & 35 & 53.14 \\
Cebada & 19 & 29.69 \\
Mora & 24 & 37.50 \\
Otros (habas, naranjillas, & 31 & 48.44 \\
y otras hortalizas) & & \\
\hline
\end{tabular}

Aspectos relacionados al sistema de producción

Cabe indicar que el $100 \%$ de los productores no realizan análisis de suelo previo a las siembras, lo cual es indicativo del desconocimiento de esta actividad como requisito importante para el éxito de la producción, demostrándose que al utilizar fertilizantes lo hacen sin previo conocimiento de los requerimientos de su terreno, es decir que posiblemente estén aplicando los nutrientes que el suelo ya posee y no aquellos de los que carece, siendo ésta una de las actividades fundamentales para el buen desarrollo del cultivo.

Referente al tipo de semilla que utilizan los productores se determinó que el $89 \%$ cultivan la variedad canario y el porcentaje restante cultivan tanto canario como panamito. La semilla que utiliza el 100\% de los productores es reciclada en sus predios, es decir, que de cada cosecha los productores acostumbran a seleccionar la semilla para la siguiente siembra, la selección la realizan aplicando criterios propios tales como: recolección de semillas provenientes de plantas vigorosas, o a su vez una vez realizada la cosecha escogen los mejores granos; esta selección deberá ser analizada y validada dado que se desconoce si los criterios de selección que están aplicando los productores garantiza o no una buena producción ya que se desconoce si la semilla presenta las condiciones adecuadas de vigor y por consiguiente si éste es uno de los factores que afectan a la producción del grano (Erazo, 2005).

\section{Mano de obra}

La mano de obra que se utiliza en todo el proceso de producción y cosecha del frijol en la comuna Panyatug en un $97 \%$ corresponde a mano de obra del productor y su familia y tan solo el 3\% corresponde a mano de obra contratada. Siendo la modalidad de trabajo horas día. Por consiguiente la mano de obra contratada generalmente es para la preparación del suelo y para 
la cosecha. Este uso generalizado de mano de obra familiar evidencia que la actividad está generando empleo para el productor y su familia coincidiendo con lo manifestado por González et al. (2007) quienes indican que en pequeñas propiedades se utiliza mano de obra para las principales actividades del cultivo.

\section{Plagas y enfermedades}

En cuanto al ataque de plagas y enfermedades y su control se determinó que un $75 \%$ de los productores han tenido ataque de enfermedades tales como: tizón (causado por la bacteria Xanthomonas axonopodis), antracnosis (causada por el hongo Colletotrichum lindemuthianum), pudrición de la raíz (causada por fusarium, Rhizoctonia Pythium y Sclerotium) y roya (causada por el hongo Uromyces phaseoli) y un $36 \%$ han tenido ataque de plagas tales como: trozadores, babosas, trips, entre otros encontrándose que el 100\% de los productores encuestados no realizan ningún tipo de control debido al desconocimiento de técnicas para su control, estas plagas y enfermedades reducen el vigor y el rendimiento de la plantación ocasionando pérdidas de hasta el $40 \%$ de la producción, llegando inclusive en algunos casos a la pérdida total de la plantación cuando existen ataques severos (Guamán et al., 2004; IICA, 2008), lo que evidencia la falta de capacitación en el manejo de este rubro productivo a los productores de esta zona del país.

\section{Fertilización}

Un 53\% de los productores encuestados indicaron que no realizan actividades de fertilización al cultivo; mientras que el $47 \%$ restante si fertiliza, de los cuales el $12.50 \%$ aplican 10-30-10 (N-P-K) en dosis de cinco sacos de $45 \mathrm{~kg} \mathrm{ha}^{-1} \mathrm{y}$, el 34\% aplica abono orgánico, de igual manera el 31\% compra el abono y el $69 \%$ lo produce, cabe indicar que aquellos productores que fertilizan lo hacen sin previo análisis de suelo es decir, desconocen las deficiencias de nutrientes en sus terrenos.

\section{Cosecha}

Relativo a la cosecha se determinó que el 100\% de los productores realizan manualmente esta actividad, utilizando para el efecto un promedio de 15 jornales con rangos que van de 5 a 20 jornales por hectárea. En cuanto al número de cosechas que se realizan en la comuna Panyatug se determinó que el 100\% de los productores realizan una sola cosecha al año, la misma que es realizada en la época de verano, cuando las vainas en un alto porcentaje están secas, esta práctica coincide con las recomendaciones de Guamán et al. (2004) quien indica que el momento oportuno para la cosecha del frijol es cuando el 90\% de las vainas están secas. Cabe indicar que el promedio del período de producción es 7 meses con rangos que van de 4 a 9 meses.

\section{Aspectos económicos}

Inversión. La inversión en una hectárea de frijol resultó ser de 1,172 USD siendo los rangos 684.40 a 1,547.00 USD con una desviación típica de 41.72 lo cual se explica debido a que los productores tienen una alta variabilidad en cuanto al manejo del cultivo.

En cuanto a la forma de financiar su producción, se determinó que el $86 \%$ lo hace con financiamiento propio y el $14 \%$ restante utiliza ambas opciones, es decir, dinero propio y préstamo a familiares.

Rendimientos. El rendimiento promedio por hectárea es $725.76 \mathrm{~kg}$ de grano seco con rangos que van de 226.80 a $1,678.32 \mathrm{~kg}$; estos datos son coherentes debido a que la producción no es igual para todos los productores.

Comercialización. La producción de frijol es vendida en un $20 \%$ en la finca a través del acopiador rural; un $64 \%$ vende en casas comerciales de la localidad, un 16\% vende tanto en la finca como en las casas comerciales (Figura 3).

Los productores al vender su producción no conocen el porcentaje de humedad del producto, ni el porcentaje de impureza del mismo; esta variable se observó en el $100 \%$ de los productores, lo cual indica que los productores desconocen totalmente la calidad de su producto, de allí que puede ser esta la causa de la variación del precio del producto y el hecho de que algunos comerciantes paguen precios por debajo de lo establecido.

Ingresos. El cultivo de frijol como actividad principal de los productores les genera ingresos anuales en el orden del $79 \%$ y el $31 \%$ de sus ingresos están dados por la venta de otros rubros tanto agrícolas (53\%) como

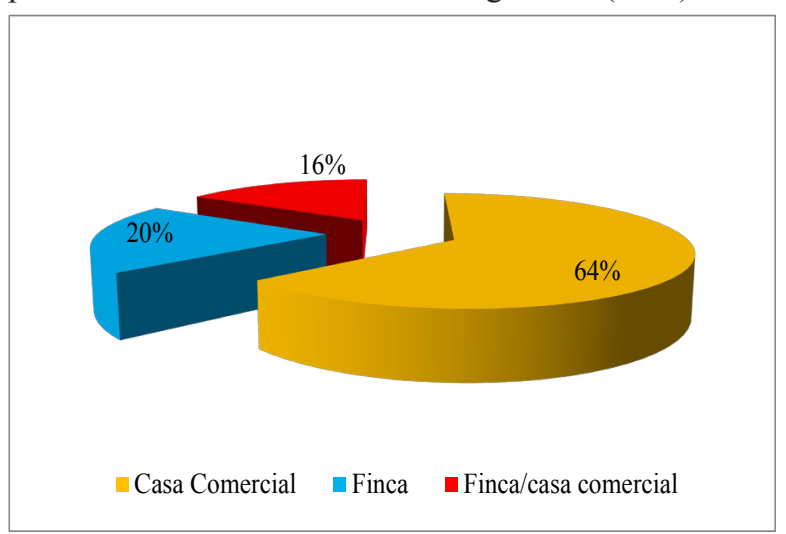

Figura 3. Destino de la venta de la producción de frijol en la comuna Panyatug 
pecuarios $(31 \%)$ o actividades extrafinca $(16 \%)$. En los rubros agrícolas destacan: venta de maíz (6\%), venta de mora $(6 \%)$, venta de trigo $(4 \%)$, venta de cebada $(3 \%)$ y venta de hortalizas $(2 \%)$. En los rubros pecuarios destacan: venta de cuyes $(8 \%)$, cerdos $(7 \%)$ y aves de corral (5\%). Las actividades extrafinca generalmente corresponden a la prestación de servicios en otras fincas o en la ciudad, como jornaleros o en actividades artesanales respectivamente, estas actividades extrafinca son realizadas en el $63 \%$ de los casos esporádicamente y el porcentaje restante las realizan durante todo el año (Figura 4).

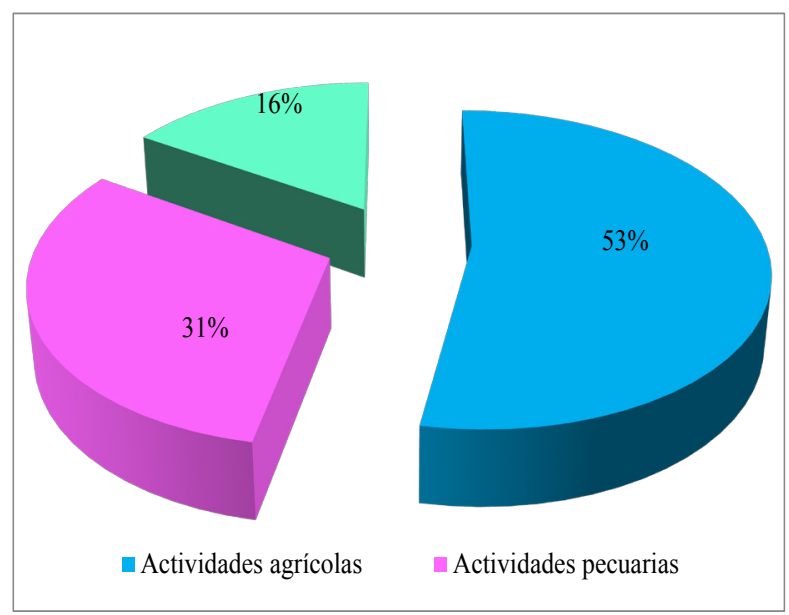

Figura 4. Otros ingresos al año que tienen los productores de frijol en la comuna Panyatug

Margen de comercialización. El margen bruto de comercialización resultó en $56 \%$ lo cual indica que por cada dólar pagado por el consumidor, 0.56 USD corresponde a la intermediación. La participación del productor (PDP) es decir la porción del precio pagado por el consumidor final que corresponde al agricultor es del $44 \%$. De acuerdo a la información recabada a intermediarios que comercializan frijol en la zona de estudio, se determinó un costo de mercadeo de 0.25 USD por quintal de frijol correspondiente a transporte, manipulación, almacenaje entre otros rubros, resultando un margen neto de comercialización de $42 \%$ (Esta información es considerada una aproximación a los costos reales, ya que los intermediarios no disponen de datos históricos sobre sus costos).

El acopiador rural tiene una participación del 3\% del precio final de frijol por concepto de acopiar el producto desde las fincas y realizar las ventas al acopiador mayorista. El mayorista tiene una participación del $22.54 \%$ del precio final de la libra de frijol por concepto de beneficiar y empacar el producto para la venta final.

Lo anterior se resume en lo siguiente: Por cada 1 dólar pagado por el consumidor corresponde al productor

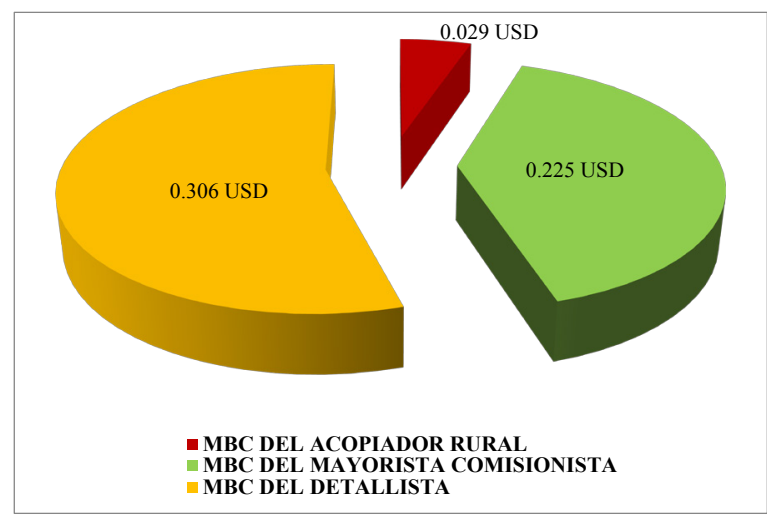

Figura 5. Margen de comercialización del frijol canario producido en la Comuna Panyatug

0.44 y a la intermediación 0.56 USD, ésta última se distribuye así: 1) para el acopiador rural 0.029 ; 2) para el mayorista comisionista $0.225 \mathrm{y}, 3$ ) para el detallista 0.306 USD (Figura 5).

Costos de producción. Con la finalidad de establecer los costos de producción de una hectárea de frijol se solicitó información referente a la estructura de los costos, encontrándose que el $100 \%$ de los productores no determinan sus costos de producción y por consiguiente desconocen su rentabilidad.

Se solicitó a los productores que enumeren las actividades que realizan desde la preparación del terreno hasta la cosecha así como los costos que incurrieron para dichas actividades, determinándose que el promedio de costos de producción corresponde a 1,172 USD (Cuadro 2). El promedio de producción de la zona es de $725.76 \mathrm{~kg}$ de frijol seco y limpio con rangos que van de 226.80 a 1,678.32. El precio promedio de venta de un kilogramo de frijol en la localidad es de 1.67 USD, siendo el rango inferior 0.66 y el superior hasta 2.20 USD el kg, lo cual demuestra que no ha habido incrementos significativos de precios respecto al 2008 difiriendo con Revista Líderes (2009) que indica sobre la existencia de una tendencia al incremento de los precios del frijol.

El mayor costo de producción corresponde al rubro de labores culturales: corte, acarreo e instalación de estacas o puntales y la deshierba manual durante el ciclo del cultivo, le sigue en importancia la fertilización, siembra y preparación del suelo con 21.12; 16.13 y $16.00 \%$, respectivamente (Figura 6).

En el análisis del destino del gasto en la explotación de frijol en la comuna Panyatug el mayor rubro (63\%) corresponde a costos de mano de obra (Figura 7), el mismo que constituye el aporte del productor y la familia, convirtiéndose de esta manera en costos de oportunidad del productor, ya que tan solo se contrata el 3\% de la 
Cuadro 2. Costo total, ingresos por venta, beneficio neto $y$ rentabilidad en la producción de frijol canario, comuna Panyatug

\begin{tabular}{lrr}
\hline \multirow{2}{*}{ Actividad/ Rubros } & \multicolumn{2}{c}{ Costo Total } \\
\cline { 2 - 3 } & \multicolumn{1}{c}{ USD } & \multicolumn{1}{c}{$\%$} \\
\hline Preparación del suelo & 187.50 & 15.99 \\
Siembra & 189.00 & 16.13 \\
Fertilización & 247.50 & 21.11 \\
Labores culturales & 330.00 & 28.16 \\
Uso del terreno & 50.00 & 4.27 \\
Cosecha & 112.50 & 9.60 \\
Transporte & 20.50 & 1.75 \\
Subtotal & $1,137.00$ & 97.01 \\
Imprevistos (3\%) & 35.00 & 2.99 \\
\hline Total Costos & $\mathbf{1 , 1 7 2 . 0 0}$ & $\mathbf{1 0 0 . 0 0}$ \\
Ingresos por venta (16 qq. & $1,216.00$ & \\
promedio) & $\mathbf{4 4 . 0 0}$ & \\
Beneficio Neto & $\mathbf{0 . 0 3 8}$ & \\
Beneficio/Costo & $\mathbf{3 . 7 5}$ & \\
Rentabilidad (\%) & & \\
\hline
\end{tabular}

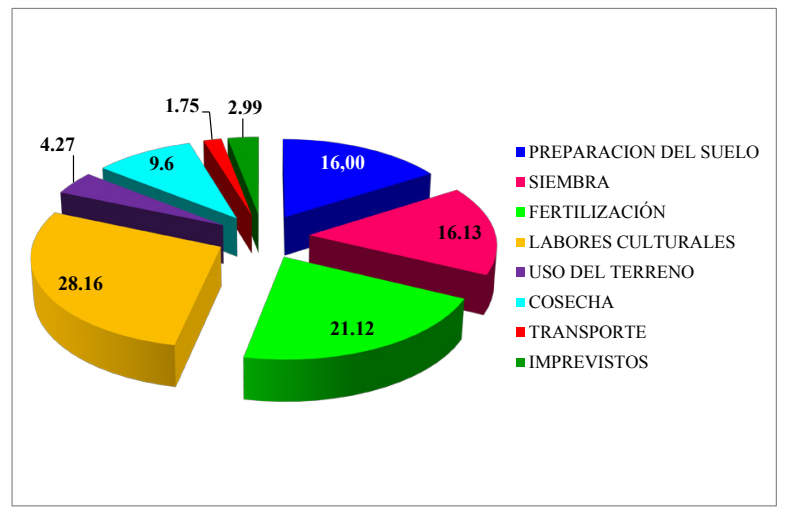

Figura 6. Estructura del costo de producción del cultivo de frijol en la Comuna Panyatug

mano de obra y el 97\% restante serían ingresos de la familia, demostrándose de esta manera que el cultivo de frijol en la Comuna Panyatug es de subsistencia pues al considerar la mano de obra de la familia se genera una rentabilidad de $97.58 \%$, mientras que sin considerar este análisis la rentabilidad corresponde al $3.75 \%$ en los siete meses del cultivo (Figura 8).

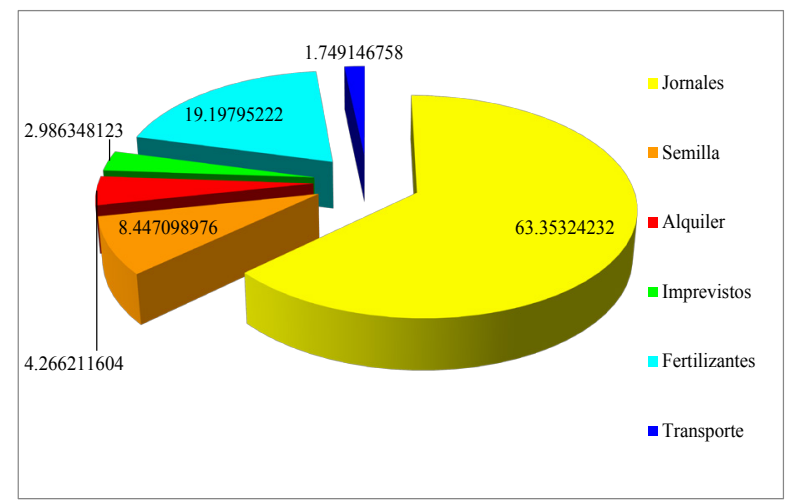

Figura 7. Destino del costo de producción del cultivo de frijol en la Comuna Panyatug

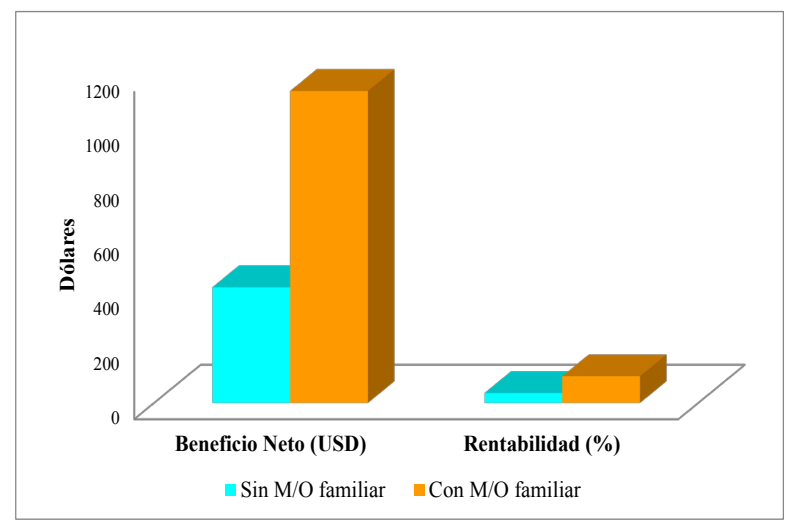

Figura 8. Beneficio Neto y rentabilidad del cultivo de frijol con y sin Mano de obra Familiar en la Comuna Panyatug

\section{Conclusiones}

T a producción de frijol en la Comuna Panyatug Udel cantón Pangua, provincia de Cotopaxi es de subsistencia con bajos niveles de uso de tecnología, sin embargo el conocimiento que tienen los productores sobre el manejo del cultivo, así como sus intenciones de continuar con el mismo permiten vislumbrar una fortaleza en esta zona del país para la actividad frejolera, ya que la mayoría de los aspectos estudiados en esta investigación son factibles de mejorar si se adoptan las tecnologías generadas por centros de investigación del país (INIAP y universidades agropecuarias) y que están siendo aplicadas en las zonas tradicionales para este cultivo.

\section{Bibliografía}

Espinosa, V., G. Rivera y L. García. 2008. Los canales y márgenes de comercialización de la leche cruda producida en sistema familiar (Estudio de caso). 
Veterinaria México 39(1).

Erazo, F. 2005. Evaluación de once variedades de fréjol (Phaseolus vulgaris L.) durante la época seca del año 2004 en la zona de Quevedo. Tesis de Ingeniero Agrónomo. Los Ríos, Ecuador. Universidad Técnica Estatal de Quevedo. $57 \mathrm{p}$

elcomercio.com. 2011. Cuatro variedades de fréjol se consumen en Ecuador. (En línea). Consultado el 19 de septiembre de 2009. Disponible en: http:// www.elcomercio.ec/agromar/variedades-frejolconsumen_0_467953228.html.

Galindo-González, G., R. Zandate-Hernández. 2006. Adopción de variedades de frijol en el noroeste de zacatecas. Terra Latinoamericana 24(1): 141-147

Garver, E., E. Falconí-Castillo, E. Peralta-Hidrovo, J. Kelly. 2008. Encuesta a productores para orientar el fitomejoramiento de frijol en Ecuador. Universidad de Costa Rica. Costa Rica Agronomía Mesoamericana, 19(1):7-18.

González, B., E. Torres, I. Giunta, J. Muñoz, G. Díaz. 2007. Respuestas Campesinas frente a la apertura comercial: Evaluación situacional de la agricultura a nivel de pequeños productores en la zona central de la Costa ecuatoriana. Los Ríos Ecuador. 112p.

Guamán, R., V. Andrade, A. Álava. 2004. Variedades mejoradas de fréjol para el litoral Ecuatoriano. Estación Experimental Boliche, Programa de Leguminosas.

IICA. (Instituto Interamericano de Cooperación para la Agricultura) 2008. Guía de identificación y manejo integrado de enfermedades del frijol en América Central. IICA. Proyecto RED SICTA, COSUDE. Managua. $32 \mathrm{p}$.

INEC. (Instituto Nacional de Estadísticas y Censos, EC). 2002. III Censo Nacional Agropecuario. Resultados nacionales y provinciales. Quito, EC. p. 66-72.

Microsoft Corporation. 1999. SPSS base 10.0. Manual del usuario. Dublín, Irlanda

Murillo, A., E. Falconí, N. Mazón, E. Peralta. 2005. Resistance sources for rust, angular leaf spot and common bacterial blight in common bean for Ecuador. Annual report of the Bean Improvement Cooperative. p 229-230.

FAO. (Organización de las Naciones Unidas para la Agricultura y la Alimentación). 2005. World crop and livestock statistics 1948-85. La Red de Información sobre Operaciones en Poscosecha (INPhO). (En línea). Consultado el 15 de mayo del 2013. Disponible en http:://www.fao.org/ag/agl/ rla128/iiiap2/capituloiii.com

Pachico, D. 1989. Trends in world common bean production. Ed S. Singh. Bean productions problems in the tropics. p 1-8.
PROFRIZA. (Proyecto Regional de Fríjol para la Zona Andina). 2000. Un cultivo ancestral avanza hacia la modernidad: tiempo de transición: 19881999. 1999. Informe final de PROFRIZA/ Cali, Colombia: Agencia Suiza para el Desarrollo y la Cooperación. $72 \mathrm{p}$.

PRONALEG-GA. (Programa Nacional de Leguminosas y Granos Andinos). 2005. Informe anual 2004: Actividades en fréjol (Phaseolus vulgaris L.). Quito-Ecuador.

Revista Líderes. 2009. Un cultivo de altura no se detiene ni con el invierno. Grupo El Comercio C.A. (En línea). Consultado el 22 de Agosto del 2009. Disponible en: http://www.revistalideres.ec/ 3334\&medio $=$ LI

Sanders, J. Y., H. Schwartz. 1980. Bean production and pest constraints in Latin America. Eds. H Schwartz y G Gálvez. Bean production problems. p. 3.

Santoyo-Cortés, H., P. Ramírez-Moreno, M. Suvedi. 2000. Manual para la evaluación de programas de desarrollo rural. Centro de Investigaciones Económicas, Sociales y Tecnológicas de la Agroindustria y la Agricultura MundialUniversidad Autónoma Chapingo-Instituto Nacional de Capacitación Rural. México, D.F.

SICA-MAG. 2000. III Censo Nacional Agropecuario. (En línea). Consultado el 22 de Agosto de 2009. Disponible en: http://www. sica.gov.ec/cadenas/ frejol/docs/frej_esp.htm

SICA-MAG-INEC. 2002. III Censo Nacional Agropecuario. Resultados Nacionales y Provinciales. Quito, EC. Volumen 1.p. 117.

Singh, S. 1999. Integrated genetic improvement. In: Common bean improvement in the twentyfirst century. Ed. S. Singh, Khrwer Academic Publishers- Dordreacth. P.1-24 y 98-99.

Trujillo, C. 2007. Estandarización del método de selección de gametos en la F1 de cruzas dobles para resistencia múltiple a enfermedades en fréjol común (Phaseolus vulgaris). Santa Catalina, Iniap. Tesis de grado Ingeniero Agropecuario. Escuela Superior Politécnica del Ejército. 78p.

Velásquez, J., P. Giraldo. 2005. Posibilidades competitivas de productos prioritarias de Antioquia frente a los acuerdos de integración y nuevos acuerdos comerciales. Gobernación de Antioquia, Departamento de Planificación- Secretaría de productividad y competitividad. Informe, $92 \mathrm{p}$.

Villegas, A., J. Carrillo, M. Jerez, B. Jarquín. 2009. Evaluación de una huerta orgánica como un modelo de producción intensiva de cultivos asociados. Revista Brasilera de Agroecología. 4(2): 35343537. 